\title{
Bayes Estimation of Change Point in Discrete Maxwell Distribution
}

\author{
Mayuri Pandya and Hardik Pandya \\ Department of Statistics, Bhavnagar University, Bhavnagar 364002, India \\ Correspondence should be addressed to Mayuri Pandya, mayuri.dave@rediffmail.com
}

Received 28 December 2010; Revised 13 May 2011; Accepted 13 May 2011

Academic Editor: Ajit K. Verma

Copyright ( $) 2011$ M. Pandya and H. Pandya. This is an open access article distributed under the Creative Commons Attribution License, which permits unrestricted use, distribution, and reproduction in any medium, provided the original work is properly cited.

A sequence of independent lifetimes $X_{1}, \ldots, X_{m}, X_{m+1}, \ldots, X_{n}$ was observed from Maxwell distribution with reliability $r_{1}(t)$ at time $t$ but later, it was found that there was a change in the system at some point of time $m$ and it is reflected in the sequence after $X_{m}$ by change in reliability $r_{2}(t)$ at time $t$. The Bayes estimators of $m, \theta_{1}, \theta_{2}$ are derived under different asymmetric loss functions. The effects of correct and wrong prior information on the Bayes estimates are studied.

\section{Introduction}

Maxwell distribution plays an important role in physics and other allied sciences. This paper introduces a discrete analogue of the Maxwell distribution, called discrete Maxwell distribution (dMax distribution). This distribution is suggested as a suitable reliability model to fit a range of discrete lifetime data.

In reliability theory many continuous lifetime models have been suggested and studied. However, it is sometimes impossible or inconvenient to measure the life length of a device, on a continuous scale. In practice, we come across situations, where lifetime of a device is considered to be a discrete random variable. For example, in an on/off switching device, the lifetime of the switch is a discrete random variable. Also, the number of voltage fluctuations which an electrical or electronic item can withstand before its failure is a discrete rv.

If the lifetimes of individuals in some population are grouped or when lifetime refers to an integral number of cycles of some sort, it may be desirable to treat it as a discrete rv. When a discrete model is used with lifetime data, it is usually a multinomial distribution, which arises because effectively continuous data have been grouped. Some situations may demand for another discrete distribution, usually over the non negative integers. Such situations are best treated individually, but generally one tries to adopt one of the standard discrete distributions.

In the last two decades, standard discrete distributions like geometric and negative binomial have been employed to model lifetime data. However, there is a need to find more plausible discrete lifetime distributions to fit to various types of lifetime data. For this purpose, popular continuous lifetime distributions can be helpful in the following manner.

The Maxwell distribution defines the speed of molecules in thermal equilibrium under some conditions as defined in statistical mechanics. For example, this distribution explains many fundamental gas properties in kinetic theory of gases; distribution of energies and moments, and so forth.

Tyagi and Bhattachary ([1], (1989b)) considered Maxwell distribution as a lifetime model for the first time. They obtained minimum variance unbiased estimator (MVUE) and Bayes estimator of the parameter and reliability function of this distribution.

Chaturvedi and Rani [2] studied generalized Maxwell distribution by introducing one more parameter and obtained classical and Bayesian estimation procedures for it. A lifetime model is specified to represent the distribution of lifetimes, and statistical inferences are made on the basis of this model. Physical systems manufacturing the items are often subject to random fluctuations. It may happen that at some point of time instability in the sequence of lifetimes 
and reliability is observed. The problem of study is when and where this change has started occurring. This is called change point inference problem. Bayesian ideas may play an important role in the study of such change point problem and has been often proposed as a valid alternative to classical estimation procedure. The monograph of Broemeling and Tsurumi [3] on structural changes, Jani and Pandya [4], Ebrahimi and Ghosh [5] and a survey by Zacks [6], Pandya and Jani [7], Pandya and Bhatt [8], Mayuri Pandya and prbha Jadav [9], Pandya and Jadav [10] are useful references. In this paper we have proposed a discrete Maxwell model to represent the distribution of lifetimes with change point $m$ and have obtained Bayes estimators of $m, \theta_{1}, \theta_{2}$.

\section{Proposed Change Point Model}

In this section we propose change point model on discrete Maxwell distribution. We also derived the Bayes estimates for the model. Let $X_{1}, X_{2}, \ldots, X_{n}(n \geq 3)$ be a sequence of random lifetimes. First $m$ of them are coming from discrete Maxwell, $\operatorname{dMax}\left(\theta_{1}\right)$. So the probability mass function is given by

$$
\begin{array}{r}
p\left(x_{i}\right)=\frac{4}{\sqrt{\pi}} \frac{1}{\theta_{1}} Q\left(x_{i}, 2, \theta_{1}\right), \quad x=0,1, \ldots, \theta_{1}>0 \\
i=1,2, \ldots, m .
\end{array}
$$

With reliability $r_{1}(t)$,

$$
r_{1}(t)=\frac{4}{\sqrt{\pi}} \theta_{1}^{-3 / 2} J\left(t, 2, \theta_{1}\right), \quad x=0,1, \ldots ; \theta_{1}>0 .
$$

Later $n-m$ observations are coming from the discrete Maxwell, $\operatorname{dMax}\left(\theta_{2}\right)$. So the probability mass function is given by

$$
\begin{array}{r}
p\left(x_{i}\right)=\frac{4}{\sqrt{\pi}} \frac{1}{\theta_{2}} Q\left(x_{i}, 2, \theta_{2}\right), \quad x=0,1, \ldots, \theta_{2}>0, \\
i=m+1, \ldots, n .
\end{array}
$$

With reliability $r_{2}(t)$,

$$
r_{2}(t)=\frac{4}{\sqrt{\pi}} \theta_{2}^{-3 / 2} J\left(t, 2, \theta_{2}\right), \quad x=0,1 \ldots ; \theta_{2}>0,
$$

where

$$
\begin{gathered}
Q\left(x, k, \theta_{1}\right)=\int_{x}^{x+1} u^{k} e^{-u^{2} / \theta_{1}} \mathrm{~d} u=J\left(x, k, \theta_{1}\right)-J\left(x+1, k, \theta_{1}\right), \\
J\left(x, k, \theta_{1}\right)=\int_{x}^{\infty} u^{k} e^{-u^{2} / \theta_{1}} \mathrm{~d} u, \\
Q\left(x, k, \theta_{2}\right)=\int_{x}^{x+1} u^{k} e^{-u^{2} / \theta_{2}} \mathrm{~d} u=J\left(x, k, \theta_{2}\right)-J\left(x+1, k, \theta_{2}\right), \\
J\left(x, k, \theta_{2}\right)=\int_{x}^{\infty} u^{k} e^{-u^{2} / \theta_{2}} \mathrm{~d} u .
\end{gathered}
$$

The likelihood function given the sample information $\underline{X}=$ $\left(\underline{X}_{1}, \underline{X}_{2}, \ldots, \underline{X}_{m}, \underline{X}_{m+1}, \ldots, \underline{X}_{n}\right)$

$$
L\left(\theta_{1}, \theta_{2}, m \mid \underline{X}\right)=\left(\frac{4}{\sqrt{\pi}}\right)^{n} \theta_{1}^{-m} G_{1}\left(m, \theta_{1}\right) \theta_{2}^{-(n-m)} G_{2}\left(m, \theta_{2}\right)
$$

where

$$
\begin{gathered}
G_{1}\left(m, \theta_{1}\right)=\prod_{i=0}^{m} Q\left(x_{i}, 2, \theta_{1}\right), \\
G_{2}\left(m, \theta_{2}\right)=\prod_{i=m+1}^{n} Q\left(x_{i}, 2, \theta_{2}\right),
\end{gathered}
$$

$Q\left(x_{i}, 2, \theta_{1}\right)$ and $Q\left(x_{i}, 2, \theta_{2}\right)$ are explained in (5a) and (5c).

\section{Bayes Estimation}

3.1. The Conjugate Analysis Using Inverted Gamma Prior Distribution. The ML method, as well as other classical approaches, is based only on the empirical information provided by the data. However, when there is some technical knowledge on the parameters of the distribution available, a Bayes procedure seems to be an attractive inferential method. The Bayes procedure is based on a posterior density, say $g\left(\theta_{1}, \theta_{2}, m \mid \underline{X}\right)$, which is proportional to the product of the likelihood function $L\left(\theta_{1}, \theta_{2}, m \mid \underline{X}\right)$ with a prior joint density, say $g\left(\theta_{1}, \theta_{2}, m\right) \mid$ representing the uncertainty on the parameters values.

We also suppose that some information on $\theta_{1}$ and $\theta_{2}$ is available and that this technical knowledge can be given in terms of prior mean values $\mu_{1}, \mu_{2}$ and variance $\sigma_{1}$ and $\sigma_{2}$, respectively. Suppose that the marginal prior density of $\theta_{1}$ and $\theta_{2}$ is the inverted Gamma with respective mean $\mu_{1}$ and $\mu_{2}$ :

$$
\begin{aligned}
& g\left(\theta_{1}\right)=\frac{a_{1}^{b_{1}}}{\Gamma b_{1}}\left(\theta_{1}\right)^{-\left(b_{1}+1\right)} e^{-a_{1} / \theta_{1}}, \\
& g\left(\theta_{2}\right)=\frac{a_{2}^{b_{2}}}{\Gamma b_{2}}\left(\theta_{2}\right)^{-\left(b_{2}+1\right)} e^{-a_{2} / \theta_{2}}, \\
& a_{i} b_{i}>0, \quad \theta_{i}>0, \quad i=1,2,
\end{aligned}
$$

where the parameters $a_{i}, b_{i} i=1,2$ are obtained by solving

$$
\begin{gathered}
b_{i}=2+\frac{\mu_{i}^{2}}{\sigma_{i}^{2}}, \\
a_{i}=\mu_{i}\left(b_{i}-1\right), \quad i=1,2 .
\end{gathered}
$$

Following Calabria and Pulcini [13] we assume the prior information to be correct if the true value of $\theta_{1}\left(\theta_{2}\right)$ is close to prior mean $\mu_{1}\left(\mu_{2}\right)$ and is assumed to be wrong if $\theta_{1}\left(\theta_{2}\right)$ is far from $\mu_{1}\left(\mu_{2}\right)$.

As in the study by Broemeling and Tsurumi [3], we suppose the marginal prior distribution of $m$ to be discrete uniform over the set $\{1,2, \ldots, n-1\}$

$$
g(m)=\frac{1}{(n-1)} \text {. }
$$


The joint prior density of $\theta_{1}, \theta_{2}, m$ is

$$
\begin{aligned}
g_{1}\left(\theta_{1}, \theta_{2}, m\right) & =\frac{1}{n-1} \frac{a_{1} b_{1}}{\Gamma b_{1}} \frac{a_{2} b_{2}}{\Gamma b_{2}} \theta_{1}^{-\left(b_{1}+1\right)} e^{-a_{1} / \theta_{1}} \theta_{2}^{-\left(b_{2}+1\right)} e^{-a_{2} / \theta_{2}} \\
& =k \theta_{1}^{-\left(b_{1}+1\right)} e^{-a_{1} / \theta_{1}} \theta_{2}^{-\left(b_{2}+1\right)} e^{-a_{2} / \theta_{2}}
\end{aligned}
$$

where

$$
k=\frac{1}{n-1} \frac{a_{1}^{b_{1}}}{\Gamma b_{1}} \frac{a_{2}^{b_{2}}}{\Gamma b_{2}} .
$$

The joint posterior density of $\theta_{1}, \theta_{2}, m$ is

$$
\begin{aligned}
g_{1}\left(\theta_{1}, \theta_{2}, m \mid \underline{x}\right)= & \frac{L\left(\theta_{1}, \theta_{2}, m \mid \underline{x}\right) g_{1}\left(\theta_{1}, \theta_{2}, m\right)}{h_{1}(\underline{x})} \\
g_{1}\left(\theta_{1}, \theta_{2}, m \mid \underline{x}\right)= & k_{2} \theta_{1}^{-\left(m+b_{1}+1\right)} e^{-a_{1} / \theta_{1}} G_{1}\left(m, \theta_{1}\right) \theta_{2}^{-\left(n-m+b_{2}+1\right)} \\
& \times e^{-a_{2} / \theta_{2}} G_{2}\left(m, \theta_{2}\right) \cdot h_{1}^{-1}(\underline{x}), \\
& k_{2}=k \cdot k_{1}=k\left(\frac{4}{\sqrt{\pi}}\right)^{n},
\end{aligned}
$$

$G_{1}\left(m, \theta_{1}\right)$ and $G_{2}\left(m, \theta_{2}\right)$ are explained in (7).

And $h_{1}(\underline{x})$ is the marginal posterior density of $\underline{X}$ :

$$
\begin{aligned}
h_{1}(\underline{x}) & =\sum_{m=1}^{n-1} \iint_{0}^{\infty} L\left(\theta_{1}, \theta_{2}, m \mid \underline{x}\right) g_{1}\left(\theta_{1}, \theta_{2}, m\right) \mathrm{d} \theta_{1} \mathrm{~d} \theta_{2} \\
& =k_{2} \sum_{m=1}^{n-1} I_{1}(m) I_{2}(m),
\end{aligned}
$$

where

$$
\begin{array}{r}
I_{1}(m)=\int_{0}^{\infty} \theta_{1}^{-\left(m+b_{1}+1\right)} e^{-a_{1} / \theta_{1}} G_{1}\left(m, \theta_{1}\right) \mathrm{d} \theta_{1}, \\
I_{2}(m)=\int_{0}^{\infty} \theta_{2}^{-\left(n-m+b_{2}+1\right)} e^{-a_{2} / \theta_{2}} G_{2}\left(m, \theta_{2}\right) \mathrm{d} \theta_{2},
\end{array}
$$

$G_{1}\left(m, \theta_{1}\right)$ and $G_{2}\left(m, \theta_{2}\right)$ are explained in (7).

Marginal posterior density of $\theta_{1}$ and of $\theta_{2}$ is obtained by integrating the joint posterior density of $\theta_{1}, \theta_{2}, m$ given in (13) with respect to $\theta_{2}$ and with respect to $\theta_{1}$, respectively, and summing over $m$,

$$
\begin{aligned}
g_{1}\left(\theta_{1} \mid \underline{x}\right)= & k_{2} \sum_{m=1}^{n-1} \theta_{1}^{-\left(m+b_{1}+1\right)} e^{-a_{1} / \theta_{1}} G_{1}\left(m, \theta_{1}\right) \\
& \times \int_{0}^{\infty} \theta_{2}^{-\left(n-m+b_{2}+1\right)} e^{-a_{2} / \theta_{2}} G_{2}\left(m, \theta_{2}\right) \mathrm{d} \theta_{2} h_{1}^{-1}(\underline{x}), \\
g_{1}\left(\theta_{2} \mid \underline{x}\right)= & k_{2} \sum_{m=1}^{n-1} \theta_{2}^{-\left(n-m+b_{2}+1\right)} e^{-a_{2} / \theta_{2}} G_{2}\left(m, \theta_{2}\right) \\
& \times \int_{0}^{\infty} \theta_{1}^{-\left(m+b_{1}+1\right)} e^{-a_{1} / \theta_{1}} G_{1}\left(m, \theta_{1}\right) \mathrm{d} \theta_{1} h_{1}^{-1}(\underline{x}),
\end{aligned}
$$

$G_{1}\left(m, \theta_{1}\right)$ and $G_{2}\left(m, \theta_{2}\right)$ are explained in $(7)$, and $h_{1}(\underline{x})$ is the same as in (15).

The marginal posterior density of change point $m$, say $g_{1}(m \mid \underline{x})$, is obtained by integrating the joint posterior density of $\theta_{1}, \theta_{2}, m(13)$ with respect to $\theta_{1}$ and $\theta_{2}$

$$
\begin{aligned}
g_{1}(m \mid \underline{x})= & \iint_{0}^{\infty} g_{1}\left(\theta_{1}, \theta_{2}, m \mid \underline{x}\right) \mathrm{d} \theta_{1} \mathrm{~d} \theta_{2} \\
= & \iint_{0}^{\infty} k_{2} \theta_{1}^{-\left(m+b_{1}+1\right)} e^{-a_{1} / \theta_{1}} G_{1}\left(m, \theta_{1}\right) \theta_{2}^{-\left(n-m+b_{2}+1\right)} \\
& \quad \times e^{-a_{2} / \theta_{2}} G_{2}\left(m, \theta_{2}\right) \mathrm{d} \theta_{1} \mathrm{~d} \theta_{2} h_{1}^{-1}(\underline{x}) \\
= & \frac{I_{1}(m) I_{2}(m)}{\sum_{m=1}^{n-1} I(m)_{1} I_{2(m)}} .
\end{aligned}
$$

The Bayes estimator of a generic parameter (or function thereof) $\alpha$, based on a squared error loss (SEL) function:

$$
L_{1}(\alpha, d)=(\alpha-d)^{2},
$$

where $d$ is a decision rule to estimate and $\alpha$, is the posterior mean.

Bayes estimator of $m$ under SEL and the inverted Gamma prior is

$$
m_{1}^{*}=\frac{\sum_{m=1}^{n-1} m I_{1}(m) I_{2}(m)}{\sum_{m=1}^{n-1} I_{1}(m) I_{2}(m)} .
$$

$I_{1}(m)$ and $I_{2}(m)$ are the same as in (16).

Other Bayes estimators of $\alpha$ based on the loss functions

$$
L_{2}(\alpha, d)=|\alpha-d|
$$

$$
L_{3}(\alpha, d)= \begin{cases}0, & \text { if }|\alpha-d|<\epsilon, \epsilon>0, \\ 1, & \text { otherwise, }\end{cases}
$$

are the posterior median and posterior mode, respectively.

3.2. Posterior Distribution Functions Using Noninformative Prior. A noninformative prior is a prior that adds no information to that contained in the empirical data. Thus, a Bayes inference based upon noninformative prior has generally a theoretical interest only, since, from an engineering view point, the Bayes approach is very attractive for it allows incorporating expert opinion or technical knowledge in the estimation procedure. Let the joint noninformative prior density of $\theta_{1}, \theta_{2}$, and $m$ be given by,

$$
g_{2}\left(\theta_{1}, \theta_{2}, m\right)=\frac{1}{(n-1) \theta_{1} \theta_{2}} .
$$

The joint posterior density using noninformative prior $g_{2}\left(\theta_{1}, \theta_{2}, m\right)$, say $g_{2}\left(\theta_{1}, \theta_{2}, m \mid \underline{x}\right)$, is

$$
\begin{aligned}
g_{2}\left(\theta_{1}, \theta_{2}, m \mid \underline{x}\right)= & k_{2} \sum_{m=1}^{n-1} \theta_{1}^{-(m+1)} e^{-1 / \theta_{1}} G_{1}\left(m, \theta_{1}\right) \\
& \times \theta_{2}^{-(n-m+1)} e^{-1 / \theta_{2}} G_{2}\left(m, \theta_{2}\right) \cdot h_{2}^{-1}(\underline{x}),
\end{aligned}
$$


where $G_{1}\left(m, \theta_{1}\right), G_{2}\left(m, \theta_{2}\right)$ and $k_{2}$ are the same as in (7) and (14) and $h_{2}(\underline{x})$ is the marginal density of $\underline{x}$ under the noninformative priors and is obtained by,

$$
\begin{aligned}
h_{2}(\underline{x}) & =\sum_{m=1}^{n-1} \iint_{0}^{\infty} L\left(\theta_{1}, \theta_{2}, m \mid \underline{x}\right) g_{2}\left(\theta_{1}, \theta_{2}, m\right) \mathrm{d} \theta_{1} \mathrm{~d} \theta_{2} \\
& =k_{2} \sum_{m=1}^{n-1} I_{3}(m) I_{4}(m),
\end{aligned}
$$

where

$$
\begin{aligned}
& I_{3}(m)=\int_{0}^{\infty} \theta_{1}^{-(m+1)} e^{-1 / \theta_{1}} G_{1}\left(m, \theta_{1}\right) \theta_{2}^{-(n-m+1)} \mathrm{d} \theta_{1}, \\
& I_{4}(m)=\int_{0}^{\infty} e^{-1 / \theta_{2}} G_{2}\left(m, \theta_{2}\right) \mathrm{d} \theta_{2} .
\end{aligned}
$$

Marginal posterior density of $\theta_{1}, \theta_{2}$ and change point $m$ under the noninformative prior (25) is obtained as

$$
\begin{aligned}
g_{2}\left(\theta_{1} \mid \underline{x}\right)= & k_{2} \sum_{m=1}^{n-1} \theta_{1}^{-(m+1)} e^{-1 / \theta_{1}} G_{1}\left(m, \theta_{1}\right) \\
& \times \int_{0}^{\infty} \theta_{2}^{-(n-m+1)} e^{-1 / \theta_{2}} G_{2}\left(m, \theta_{2}\right) \mathrm{d} \theta_{2} \cdot h_{2}^{-1}(\underline{x}), \\
g_{2}\left(\theta_{2} \mid \underline{x}\right)= & k_{2} \sum_{m=1}^{n-1} e^{-1 / \theta_{2}} G_{2}\left(m, \theta_{2}\right) \theta_{2}^{-(n-m+1)} \\
& \times \int_{0}^{\infty} \theta_{1}^{-(m+1)} e^{-1 / \theta_{1}} G_{1}\left(m, \theta_{1}\right) \mathrm{d} \theta_{1} \cdot h_{2}^{-1}(\underline{x}),
\end{aligned}
$$

where $G_{1}\left(m, \theta_{1}\right), G_{2}\left(m, \theta_{2}\right)$, and $k_{2}$ are same as in (7) and (14). $h_{2}(\underline{x})$ is same as in (26).

The marginal posterior density of change point $m$ is, say $g_{2}(m \mid \underline{x})$ is obtained by integrating the joint posterior density of $\theta_{1}, \theta_{2}, m(25)$ with respect to $\theta_{1}$ and $\theta_{2}$,

$$
\begin{aligned}
g_{2}(m \mid \underline{x})= & \iint_{0}^{\infty} g_{2}\left(\theta_{1}, \theta_{2}, m \mid \underline{x}\right) \mathrm{d} \theta_{1} \mathrm{~d} \theta_{2} \\
= & \iint_{0}^{\infty} k_{2} \sum_{m=1}^{n-1} \theta_{1}^{-(m+1)} e^{-1 / \theta_{1}} G_{1}\left(m, \theta_{1}\right) \theta_{2}^{-(n-m+1)} \\
& \times e^{-1 / \theta_{2}} G_{2}\left(m, \theta_{2}\right) \cdot h_{2}^{-1}(\underline{x}) \mathrm{d} \theta_{1} \mathrm{~d} \theta_{2} \\
= & \frac{I_{3}(m) I_{4}(m)}{\sum_{m=1}^{n-1} I_{3}(m) I_{4}(m)}
\end{aligned}
$$

where $I_{3}(m)$ and $I_{4}(m)$ are the same as in (27).

Bayes estimator of $m$ under SEL and noninformative prior is

$$
m_{2}^{*}=\frac{\sum_{m=1}^{n-1} m I_{3}(m) I_{4}(m)}{\sum_{m=1}^{n-1} I_{3}(m) I_{4}(m)} .
$$

\section{Bayes Estimates of Change Point and Other Parameters under Asymmetric Loss Functions}

The loss function $L(\alpha, d)$ provides a measure of the financial consequences arising from a wrong decision rule $d$ to estimate an unknown quantity $\alpha$. The choice of the appropriate loss function depends on financial considerations only and is independent of the estimation procedure used. The use of symmetric loss function was found to be generally inappropriate, since, for example, an overestimation of the reliability function is usually much more serious than an underestimation.

In this section, we derive Bayes estimators of change point $m$ under different asymmetric loss functions using both prior considerations explained in Sections 3.1 and 3.2. A useful asymmetric loss, known as the Linex loss function was introduced by Varian [12]. Under the assumption that the minimal loss occurs at $d$, the Linex loss function can be expressed as

$$
L_{4}(\alpha, d)=\exp \left[q_{1}(d-\alpha)\right]-q_{1}(d-\alpha)-1, \quad q_{1} \neq 0 .
$$

The sign of the shape parameter $q_{1}$ reflects the deviation of the asymmetry, $q_{1}>0$, if over, estimation is more serious than underestimation, and vice versa, and the magnitude of $q_{1}$ reflects the degree of asymmetry.

The posterior expectation of the Linex loss function is

$$
E_{\alpha}\left\{L_{4}(\alpha, d)\right\}=\exp \left(q_{1} d\right) E_{\alpha}\left\{\exp \left(-q_{1} \alpha\right)\right\}-q_{1}(d-E\{\alpha\})-I,
$$

where $E_{\alpha}\{f(a)\}$ denotes the expectation of $f(a)$ with respect to the posterior density $g(\alpha \mid \underline{x})$. The Bayes estimate $a_{l}^{*}$ is the value of $d$ that minimizes $E_{\alpha}\left\{l_{4}(\alpha, d)\right\}$

$$
a_{l}^{*}=-\frac{1}{q_{1}} \ln \left[E_{\alpha}\left\{\exp \left(-q_{1} \alpha\right)\right\}\right]
$$

provided that $E_{\alpha}\left\{\exp \left(-q_{1} \alpha\right)\right\}$ exists and is finite.

Minimizing expected loss function $E_{m}\left[L_{4}(m, d)\right]$ and using posterior distributions (20) and (30), we get the Bayes estimators of $m$ using Linex loss function, respectively, as

$$
\begin{aligned}
& m_{L}^{*}=-\frac{1}{q_{1}} \ln \left[\frac{\sum_{m=1}^{n-1} e^{-m q_{1}} I_{1}(m) I_{2}(m)}{\sum_{m=1}^{n-1} I_{1}(m) I_{2}(m)}\right], \\
& m_{L}^{* *}=-\frac{1}{q_{1}} \ln \left[\frac{\sum_{m=1}^{n-1} e^{-m q_{1}} I_{3}(m) I_{4}(m)}{\sum_{m=1}^{n-1} I_{3}(m) I_{4}(m)}\right],
\end{aligned}
$$

where $I_{1}(m) I_{2}(m)$ and $I_{3}(m) I_{4}(m)$ are the same as in (16) and (27). 
Minimizing expected loss function $E_{\theta_{1}}\left[L_{4}\left(\theta_{1}, d\right)\right]$ and using posterior distributions (18) and (28), we get the Bayes estimators of $\theta_{1}$ using Linex loss function, respectively, as

$$
\begin{aligned}
\theta_{1 L}^{*}=-\frac{1}{q_{1}} \ln \left[k_{2} \sum_{m=1}^{n-1} \int_{0}^{\infty} \theta_{2}^{-\left(n-m+b_{2}+1\right)} e^{-a_{2} / \theta_{2}} G_{2}\left(m, \theta_{2}\right) \mathrm{d} \theta_{2}\right. \\
\quad \times \int_{0}^{\infty} \theta_{1}^{-\left(m+b_{1}+1\right)} e^{-a_{1} / \theta_{1}-\theta_{1} q_{1}} \\
\left.\times G_{1}\left(m, \theta_{1}\right) \mathrm{d} \theta_{1} h_{1}^{-1}(\underline{x})\right], \\
\theta_{1 L}^{* *}=\frac{1}{q_{1}} \ln \left[\int_{0}^{\infty} k_{2} \sum_{m=1}^{n-1} \theta_{1}^{-(m+1)} e^{-1 / \theta_{1}-\theta_{1} q_{1}} G_{1}\left(m, \theta_{1}\right) \mathrm{d} \theta_{1}\right. \\
\left.\times \int_{0}^{\infty} \theta_{2}^{-(n-m+1)} e^{-1 / \theta_{2}} G_{2}\left(m, \theta_{2}\right) \mathrm{d} \theta_{2} \cdot h_{2}^{-1}(\underline{x})\right],
\end{aligned}
$$

where $G_{1}\left(m, \theta_{1}\right), G_{2}\left(m, \theta_{2}\right)$, and $k_{2}$ are the same as in (7) and (14). and $h_{1}(\underline{x})$ and $h_{2}(\underline{x})$ are the same as in (15) and (26).

Minimizing expected loss function $E_{\theta_{2}}\left[L_{4}\left(\theta_{2}, d\right)\right]$ and using posterior distribution (19) and (29), we get the Bayes estimators of $\theta_{2}$ using Linex loss function, respectively, as

$$
\begin{aligned}
\theta_{2 L}^{*}=\frac{1}{q_{1}} \ln \left[k_{2} \sum_{m=1}^{n-1} \int_{0}^{\infty} \theta_{1}^{-\left(m+b_{1}+1\right)} e^{-a_{1} / \theta_{1}} G_{1}\left(m, \theta_{1}\right) \mathrm{d} \theta_{1}\right. \\
\times \int_{0}^{\infty} \theta_{2}^{-\left(n-m+b_{2}+1\right)} e^{-a_{2} / \theta_{2}-\theta_{2} q_{1}} \\
\left.\times G_{2}\left(m, \theta_{2}\right) \mathrm{d} \theta_{2} h_{1}^{-1}(\underline{x})\right], \\
\theta_{2 L}^{* *}=-\frac{1}{q_{1}} \ln \left[k_{2} \int_{0}^{\infty} e^{-1 / \theta_{2}-\theta_{2} q_{1}} G_{2}\left(m, \theta_{2}\right) \theta_{2}^{-(n-m+1)} \mathrm{d} \theta_{2}\right. \\
\left.\times \int_{0}^{\infty} \theta_{1}^{-(m+1)} e^{-1 / \theta_{1}} G_{1}\left(m, \theta_{1}\right) \mathrm{d} \theta_{1} \cdot h_{2}^{-1}(\underline{x})\right],
\end{aligned}
$$

where $G_{1}\left(m, \theta_{1}\right), G_{2}\left(m, \theta_{2}\right)$, and $k_{2}$ are the same as in (7) and (14) and $h_{1}(\underline{x})$ and $h_{2}(\underline{x})$ are the same as in (15) and (26).

Another loss function, called General Entropy (GE) loss function, proposed by Calabria and Pulcini [11], is given by,

$$
L_{5}(\alpha, d)=\left(\frac{d}{\alpha}\right)^{q_{3}}-q_{3} \ln \left(\frac{d}{\alpha}\right)-I
$$

The Bayes estimate $\alpha_{E}^{*}$ is the value of $d$ that minimizes $E_{\alpha}\left[L_{5}(\alpha, d)\right]$ :

$$
\alpha_{E}^{*}=\left[E_{\alpha}\left(\alpha^{-q_{3}}\right)\right]^{-1 / q_{3}},
$$

provided that $E_{\alpha}\left(\alpha^{-q_{3}}\right)$ exists and is finite.
Minimizing expectation $E_{m}\left[L_{5}(m, d)\right]$ and using posterior distributions (20) and (30), we get the Bayes estimators $m_{E}^{*}, m_{E}^{* *}$ of $m$, respectively, as

$$
\begin{gathered}
m_{E}^{*}=\left[E_{m}\left[m^{-q_{3}}\right]\right]^{-1 / q_{3}}=\left[\frac{\sum_{m=1}^{n-1} \mathrm{~m}^{-q_{3}} I_{1}(m) I_{2}(m)}{\sum_{m=1}^{n-1} I_{1}(m) I_{2}(m)}\right]^{-1 / q_{3}}, \\
m_{E}^{* *}=\left[\frac{\sum_{m=1}^{n-1} m^{-q_{3}} I_{3}(m) I_{4}(m)}{\sum_{m=1}^{n-1} I_{3}(m) I_{4}(m)}\right]^{-1 / q_{3}},
\end{gathered}
$$

where $I_{1}(m) I_{2}(m)$ and $I_{3}(m) I_{4}(m)$ are the same as in (16) and (27).

Minimizing expected loss function $E_{\theta_{1}}\left[L_{5}\left(\theta_{1}, d\right)\right]$ and using posterior distributions (18) and (28), we get the Bayes estimates of $\theta_{1}$ using General Entropy loss function and informative and noninformative prior, respectively, as

$$
\begin{aligned}
\theta_{1 E}^{*}= & {\left[k_{2} \sum_{m=1}^{n-1} \int_{0}^{\infty} \theta_{2}^{-\left(n-m+b_{2}+q_{3}+1\right)} e^{-a_{2} / \theta_{2}} G_{2}\left(m, \theta_{2}\right) \mathrm{d} \theta_{2}\right.} \\
& \left.\times \int_{0}^{\infty} \theta_{1}^{-\left(m+b_{1}+1\right)} e^{-a_{1} / \theta_{1}} G_{1}\left(m, \theta_{1}\right) \mathrm{d} \theta_{1} h_{1}^{-1}(\underline{x})\right]^{-1 / q_{3}}, \\
\theta_{1 E}^{* *}= & {\left[\int_{0}^{\infty} k_{2} \sum_{m=1}^{n-1} \theta_{1}^{-\left(m+q_{3}+1\right)} e^{-1 / \theta_{1}} G_{1}\left(m, \theta_{1}\right) \mathrm{d} \theta_{1}\right.} \\
& \left.\times \int_{0}^{\infty} \theta_{2}^{-(n-m+1)} e^{-1 / \theta_{2}} G_{2}\left(m, \theta_{2}\right) \mathrm{d} \theta_{2} \cdot h_{2}^{-1}(\underline{x})\right]^{-1 / q_{3}},
\end{aligned}
$$

where $G_{1}\left(m, \theta_{1}\right), G_{2}\left(m, \theta_{2}\right)$ and $k_{2}$ are same as in (7) and (14). $h_{1}(\underline{x})$ and $h_{2}(\underline{x})$ are same as in (15) and (26).

Minimizing expected loss function $E_{\theta_{2}}\left[L_{5}\left(\theta_{2}, d\right)\right]$ and using posterior distributions (19) and (29), we get the Bayes estimates of $\theta_{2}$ using General Entropy loss function as

$$
\begin{gathered}
\theta_{2 E}^{*}=\left[k_{2} \sum_{m=1}^{n-1} \int_{0}^{\infty} \theta_{1}^{-\left(m+b_{1}+1\right)} e^{-a_{1} / \theta_{1}} G_{1}\left(m, \theta_{1}\right) \mathrm{d} \theta_{1}\right. \\
\times \int_{0}^{\infty} \theta_{2}^{-\left(n-m+b_{2}+q_{3}+1\right)} e^{-a_{2} / \theta_{2}} \\
\left.\quad \times G_{2}\left(m, \theta_{2}\right) \mathrm{d} \theta_{2} h_{1}^{-1}(\underline{x})\right]^{-1 / q_{3}}, \\
\theta_{2 E}^{* *}=\left[k_{2} \int_{0}^{\infty} e^{-1 / \theta_{2}} G_{2}\left(m, \theta_{2}\right) \theta_{2}^{-\left(n-m+q_{3}+1\right)} \mathrm{d} \theta_{2}\right. \\
\left.\quad \times \int_{0}^{\infty} \theta_{1}^{-(m+1)} e^{-1 / \theta_{1}} G_{1}\left(m, \theta_{1}\right) \mathrm{d} \theta_{1} \cdot h_{2}^{-1}(\underline{x})\right]^{-1 / q_{3}},
\end{gathered}
$$

where, $G_{1}\left(m, \theta_{1}\right), G_{2}\left(m, \theta_{2}\right)$ and $k_{2}$ are same as in (7) and (14) and $h_{1}(\underline{x})$ and $h_{2}(\underline{x})$ are the same as in (15) and (26).

Remark 1. Putting $q_{3}=-1$ in (40), (41), and (42), we get the Bayes estimators of $m, \theta_{1}$, and $\theta_{2}$, posterior means under the squared error loss. 
TABLe 1: Generated samples from dMax distribution.

\begin{tabular}{lccccc}
\hline Sample no. & $N$ & $M$ & $\begin{array}{c}\theta_{1}=1.0 \\
\text { (first } m \text { observation) }\end{array}$ & $\begin{array}{c}\theta_{2}=0.5 \\
\text { (last }(n-m) \text { observation) }\end{array}$ & $R_{1}(t)$ \\
\hline 1 & 30 & 15 & $0 \times 5,1 \times 10$ & $0 \times 11,1 \times 4$ & 0.0460 \\
2 & 50 & 25 & $0 \times 9,1 \times 16$ & $0 \times 18,1 \times 7$ & 0.0011 \\
3 & 50 & 35 & $0 \times 11,1 \times 24$ & $0 \times 25,1 \times 10$ & $\theta_{2}=2.0$ \\
& & & $\theta_{1}=5.0$ & $0,1 \times 10,2 \times 4$ & 0.8495 \\
5 & 30 & 15 & $1 \times 6,2 \times 4,3 \times 2,4 \times 2$ & $0 \times 3,1 \times 16,2 \times 6$ & 0.6594 \\
6 & 50 & 25 & $0,1 \times 9,2 \times 6,3 \times 6,4 \times 3$ & $0 \times 5,1 \times 21,2 \times 8,3$ & \\
\hline
\end{tabular}

TABLE 2: Bayes estimate of $m, \theta_{1}$, and $\theta_{2}$ under SEL.

\begin{tabular}{|c|c|c|c|c|}
\hline \multirow[b]{2}{*}{ Sample no. } & \multirow[b]{2}{*}{$N$} & \multirow{2}{*}{ Bayes estimates of $m$ (posterior mean) } & \multicolumn{2}{|c|}{ Bayes Estimates of $\theta_{1}$ and $\theta_{2}$ (posterior mean) } \\
\hline & & & $\theta_{1}^{*}$ & $\theta_{2}^{*}$ \\
\hline 1 & 30 & 15 & 1.0 & 0.5 \\
\hline 2 & 50 & 25 & 1.0 & 0.5 \\
\hline 3 & 50 & 35 & 1.0 & 0.5 \\
\hline 4 & 30 & 15 & 5.0 & 2.0 \\
\hline 5 & 50 & 25 & 5.0 & 2.0 \\
\hline 6 & 50 & 35 & 5.0 & 2.0 \\
\hline
\end{tabular}

Note that, for $q_{3}=-1$, the GE loss function reduces to the squared error loss function.

\section{Numerical Study}

We have generated 30 random observations from dmax distribution involving change point discussed in Section 2. The first 15 observations from dMax with $\theta_{1}=1.0$ and at $t=5.0, R_{1 t}=0.0460$ and next 15 observations from dMax distribution with $\theta_{2}=0.5$ and $R_{2 t}=0.0011, \theta_{1}$ and $\theta_{2}$ themselves were random observation from inverted Gamma prior distributions with prior means $\mu_{1}=1.0, \mu_{2}=0.5$ and variance $\sigma_{1}=1.0$ and $\sigma_{2}=1.0$, respectively, resulting in $a_{1}=2.0, b_{1}=3.0$, and $a_{2}=.5, b_{2}=.75$. These observations are given in Table 1 first raw.

We also compute the Bayes estimators $m_{L}^{*}, m_{E}^{*}$ of $m$, $\theta_{1 E}^{*}, \theta_{1 L}^{*}$ and $\theta_{2 E}^{*}, \theta_{2 L}^{*}$ of $\theta_{1}$ and $\theta_{2}$ using the results given in Section 4 for the data given in Table 1 and for different values of shape parameter $q_{1}$ and $q_{3}$. The results are shown in Tables 3 and 4 .

We have generated 6 random samples from discrete Maxwell distribution involving change point discussed in Section 2 with $n=30,50,50, m=15,25,35$, and $\theta_{1}=$ 1.0, 5.0 and $\theta_{2}=0.5$ and 2.0. As explained in Section 3.1, $\theta_{1}$ and $\theta_{2}$ themselves were random observations from inverted Gamma prior distributions with prior means $\mu_{1}, \mu_{2}$, respectively. These observations are given in Table 1 . We have calculated posterior means of $m, \theta_{1}$, and $\theta_{2}$ under both the prior for all samples, and the results are shown in Table 2.

Table 3 shows that for small values of $|q|, q_{1}=0.09$, $0.2,0.1$ Linex loss function is almost symmetric and nearly quadratic and the values of the Bayes estimate under such a loss is not far from the posterior mean. Table 3 also shows that, for $q_{1}=1.5,1.2$, Bayes estimates are less than actual value of $m=15$.

For $q_{1}=q_{3}=-1,-2$, Bayes estimates are quite large than actual value $m=15$. It can be seen from Tables 3 and 4 that the negative sign of shape parameter of loss functions reflecting underestimation is more serious than overestimation. Thus, problem of underestimation can be solved by taking the value of shape parameters of Linex and General Entropy loss functions negative.

Table 4 shows that, for small values of $|q|, q_{3}=0.09,0.2$, 0.1 General Entropy loss function, the values of the Bayes estimate under such a loss is not far from the posterior mean. Table 4 also shows that, for $q_{3}=1.5,1.2$, Bayes estimates are less than actual value of $m=15$.

It can be seen from Tables 3 and 4 that positive sign of shape parameter of loss functions reflecting overestimation is more serious than under estimation. Thus, problem of over estimation can be solved by taking the value of shape parameter of Linex and General Entropy loss functions positive and high.

5.1. Sensitivity of Bayes Estimates. In this section, we study the sensitivity of the Bayes estimator, obtained in Section 3 with respect to change in the prior of parameters. The means $\mu_{1}$ and $\mu_{2}$ of inverted Gamma prior on $\theta_{1}$ and $\theta_{2}$ have been used as prior information in computing the parameters $a_{1}$, $a_{2}, b_{1}$, and $b_{2}$ of the prior. We have computed posterior mean $m^{*}$ for the data given in Table 1, considering different sets of values of $\left(\mu_{1}, \mu_{2}\right)$. Following Calabria and Pulcini [13], we also assume the prior information to be correct if the true value of $\theta_{1}$ and $\theta_{2}$ is closed to prior mean $\mu_{1}\left(\mu_{2}\right)$ and 
TABle 3: The Bayes estimates using Linex loss function. ( $n=30$, $m=15, \theta_{1}=1.0, \theta_{2}=0.5$ ).

\begin{tabular}{lcccc}
\hline & $q_{1}$ & $m_{L}^{*}$ & $\theta_{1 L}^{*}$ & $\theta_{2 L}^{*}$ \\
\hline \multirow{3}{*}{ Informative prior } & 0.09 & 15 & 1.0 & 0.53 \\
& 0.10 & 15 & 1.0 & 0.52 \\
& 0.20 & 15 & 1.0 & 0.51 \\
\hline \multirow{4}{*}{ Noninformative Prior } & 1.2 & 14 & 0.97 & 0.48 \\
& 1.5 & 13 & 0.90 & 0.44 \\
& -1.0 & 16 & 1.5 & 0.57 \\
& -2.0 & 17 & 1.7 & 0.59 \\
\hline \multirow{6}{*}{} & 0.09 & 14 & 0.93 & 0.54 \\
& 0.10 & 14 & 0.92 & 0.51 \\
& 0.20 & 14 & 0.91 & 0.50 \\
\hline & 1.2 & 13 & 0.85 & 0.46 \\
& 1.5 & 12 & 0.82 & 0.41 \\
& -1.0 & 16 & 1.5 & 0.56 \\
& -2.0 & 17 & 1.7 & 0.57 \\
\hline
\end{tabular}

TABle 4: The Bayes estimates using General Entropy loss function. $\left(n=30, m=15, \theta_{1}=1.0, \theta_{2}=0.5\right)$.

\begin{tabular}{lcccc}
\hline \multirow{3}{*}{ Informative Prior } & $q_{3}$ & $\theta_{1 E}^{*}$ & $m_{E}^{*}$ & $\theta_{2 E}^{*}$ \\
\hline \multirow{4}{*}{ Noninformative Prior } & 0.09 & 1.3 & 15 & 0.53 \\
& 0.10 & 1.3 & 15 & 0.51 \\
& 0.20 & 1.2 & 15 & 0.50 \\
\hline & 1.20 & 1.0 & 13 & 0.47 \\
& 1.50 & 0.93 & 12 & 0.45 \\
& -1.0 & 1.5 & 16 & 0.55 \\
& -2.0 & 1.7 & 17 & 0.58 \\
\hline \multirow{5}{*}{} & 0.09 & 1.2 & 14 & 0.53 \\
& 0.20 & 1.2 & 14 & 0.53 \\
& 1.2 & 0.90 & 12 & 0.47 \\
& 1.5 & 0.84 & 11 & 0.44 \\
& -1.0 & 1.4 & 17 & 0.58 \\
& -2.0 & 1.8 & 19 & 0.61 \\
\hline
\end{tabular}

is assumed to be wrong if $\theta_{1}$ and $\theta_{2}$ are far from $\mu_{1}\left(\mu_{2}\right)$. We observed that the posterior mean $m^{*}$ appears to be robust with respect to the correct choice of the prior density of $\theta_{1}\left(\theta_{2}\right)$ and a wrong choice of the prior density of $\theta_{2}\left(\theta_{1}\right)$.

This can be seen from Tables 5, 6, and 7 .

Table 5 shows that, when prior mean $\mu_{1}=1=$ actual value of $\theta_{1}, \mu_{2}=0.3$ and 0.7 (far from true value of $\theta_{2}=0.5$ ), it means correct choice of prior of $\theta_{1}$ and wrong choice of prior of $\theta_{2}$, the values of Bayes estimator posterior mean remains the same and is 15. It gives correct estimation of $m$. Thus, posterior mean is not sensitive with wrong choice of prior density of $\theta_{2}\left(\theta_{1}\right)$ and correct choice of prior of $\theta_{1}\left(\theta_{2}\right)$.

\section{Simulation Study}

In Section 5, we have obtained Bayes estimates of $m$ on the basis of the generated data given in Table 1 for given
TABle 5: Bayes estimate of $m$ for Sample 1.

\begin{tabular}{llc}
\hline$\mu_{1}$ & $\mu_{2}$ & $m^{*}$ \\
\hline 1.0 & 0.3 & 15 \\
1.0 & 0.5 & 15 \\
1.0 & 0.7 & 15 \\
0.7 & 0.5 & 15 \\
0.9 & 0.5 & 15 \\
1.4 & 0.5 & 15 \\
\hline
\end{tabular}

TABle 6: Bayes estimate of $m$ for Sample 5.

\begin{tabular}{lll}
\hline$\mu_{1}$ & $\mu_{2}$ & $m^{*}$ \\
\hline 5.0 & 1.0 & 25 \\
5.0 & 3.0 & 25 \\
5.0 & 4.0 & 25 \\
4.0 & 2.0 & 25 \\
6.0 & 2.0 & 25 \\
6.5 & 2.0 & 25 \\
\hline
\end{tabular}

Table 7: Bayes Estimate of $m$ for Sample 6.

\begin{tabular}{lll}
\hline$\mu_{1}$ & $\mu_{2}$ & $m^{*}$ \\
\hline 1.0 & 0.3 & 35 \\
1.0 & 0.5 & 35 \\
1.0 & 0.7 & 35 \\
0.7 & 0.5 & 35 \\
0.9 & 0.5 & 35 \\
1.4 & 0.5 & 35 \\
\hline
\end{tabular}

values of parameters. To justify the results, we have generated 10,000 different random samples with $m=10, n=30,50$, $\theta_{1}=1, \theta_{2}=0.5$ and obtained the frequency distributions of posterior mean $m_{L}^{*}, m_{E}^{*}$ of $m$ with the correct prior consideration. The result is shown in Table 8 . The value of shape parameter of the General Entropy loss and Linex loss used in simulation study for change point is taken as 0.1 . We have also simulated several dMax samples with $m=$ $15,25,35 ; n=30,50$, and $\theta_{1}=0.15,0.11,0.10 ; \theta_{2}=$ $0.55,0.45,0.35$. For each $m, n, \theta_{1}$, and $\theta_{2} 1000$ pseudorandom samples have been simulated and Bayes estimators of change point $m$ using $q_{1}=q_{3}=0.9$ have been computed for same value of $a_{1}, a_{2}$ and for different prior means $\mu_{1}$ and $\mu_{2}$. We observed that the posterior mean $m^{*}$ appears to be robust with respect to the correct choice of the prior density of $\theta_{1}\left(\theta_{2}\right)$ and a wrong choice of the prior density of $\theta_{2}\left(\theta_{1}\right)$.for each combination of prior means $\mu_{1}$ and $\mu_{2}$.

The value of Bayes estimator of change point $m$, based on Linex loss and general Entropy loss, using $q_{1}=q_{3}=0.9$ is 10.

Table 8 leads to conclusion that performance of posterior means has better performance than that of $m_{L}^{*}, m_{E}^{*}$, of change point. $78 \%$ values of posterior mean are closed to actual value of change point with correct choice of prior. $65 \%$ values of $m_{L}^{*}$ are closed to actual value of change point with correct 
TABle 8: Frequency distributions of the Bayes estimates of the change point.

\begin{tabular}{cccc}
\hline Bayes estimate & \multicolumn{3}{c}{$\begin{array}{c}\text { \% frequency for } \\
09-11\end{array}$} \\
\hline Posterior mean & 12 & 78 & $12-20$ \\
$m_{L}^{*}$ & 20 & 65 & 10 \\
$m_{E}^{*}$ & 22 & 66 & 15 \\
\hline
\end{tabular}

choice of prior. $66 \%$ values of $m_{E}^{*}$ are close to correct values of change point with correct prior considerations.

\section{Acknowledgment}

The authors would like to thank the editor and the referee for their valuable suggestions which improved the earlier version of the paper.

\section{References}

[1] R. K. Tyagi and S. K. Bhattacharya, "Bayes estimation of the Maxwell's velocity distribution function," Statistica, vol. 29, no. 4, pp. 563-567, 1989.

[2] A. Chaturvedi and U. Rani, "Classical and Bayesian Reliability estimation of the generalized Maxwell failure distribution," Journal of Statistical Research, vol. 32, pp. 113-120, 1998.

[3] L. D. Broemeling and H. Tsurumi, Econometrics and Structural Change, Marcel Dekker, New York, NY ,USA, 1987.

[4] P. N. Jani and M. Pandya, "Bayes estimation of shift point in left truncated exponential sequence," Communications in Statistics, vol. 28, no. 11, pp. 2623-2639, 1999.

[5] N. Ebrahimi and S. K. Ghosh, "Ch. 31. Bayesian and frequentist methods in change-point problems," in Handbook of Statistics, N. Balakrishna and C. R. Rao, Eds., vol. 20, pp. 777-787, 2001.

[6] S. Zacks, "Survey of classical and Bayesian approaches to the change point problem: fixed sample and sequential procedures for testing and estimation," in Recent Advances in Statistics. Herman Chernoff Best Shrift, pp. 245-269, Academic Press, New York, NY, USA, 1983.

[7] M. Pandya and P. N. Jani, "Bayesian estimation of change point in inverse weibull sequence," Communications in Statistics, vol. 35, no. 12, pp. 2223-2237, 2006.

[8] M. Pandya and S. Bhatt, "Bayesian estimation of shift point in Weibull distribution," Journal of the Indian Statistical Association, vol. 45, no. 1, pp. 67-80, 2007.

[9] M. Pandya and P. Jadav, "Bayesian estimation of change point in inverse Weibull distribution," IAPQR Transactions, vol. 33, no. 1, pp. 1-23, 2008.

[10] M. Pandya and P. Jadav, "Bayesian estimation of change point in mixture of left truncated exponential and degenerate distribution," Communication in Statistics, vol. 39, no. 15, pp. 2742-2742, 2010.

[11] R. Calabria and G. Pulcini, "Bayes credibility intervals for the left-truncated exponential distribution," Microelectronics Reliability, vol. 34, no. 12, pp. 1897-1907, 1994.

[12] H. R. Varian, "A Bayesian approach to real estate assessment," in Studies in Bayesian Econometrics and Statistics in Honor of Leonard J. Savage, Feigner and A. Zellner, Eds., pp. 195-208, North Holland, Amsterdam, The Netherlands, 1975.
[13] R. Calabria and G. Pulcini, "Point estimation under asymmetric loss functions for left-truncated exponential samples," Communications in Statistics, vol. 25, no. 3, pp. 585-600, 1996. 

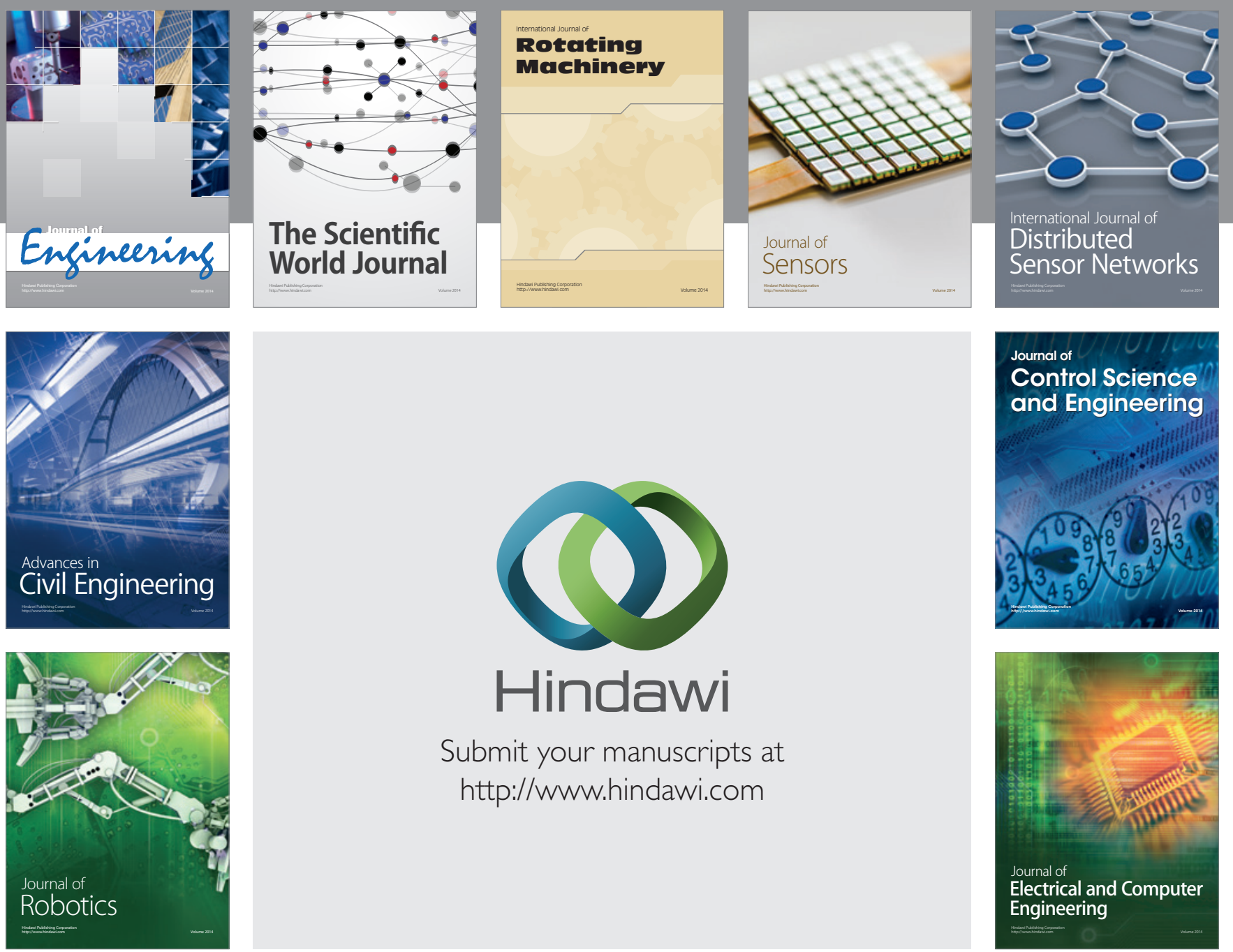

Submit your manuscripts at

http://www.hindawi.com
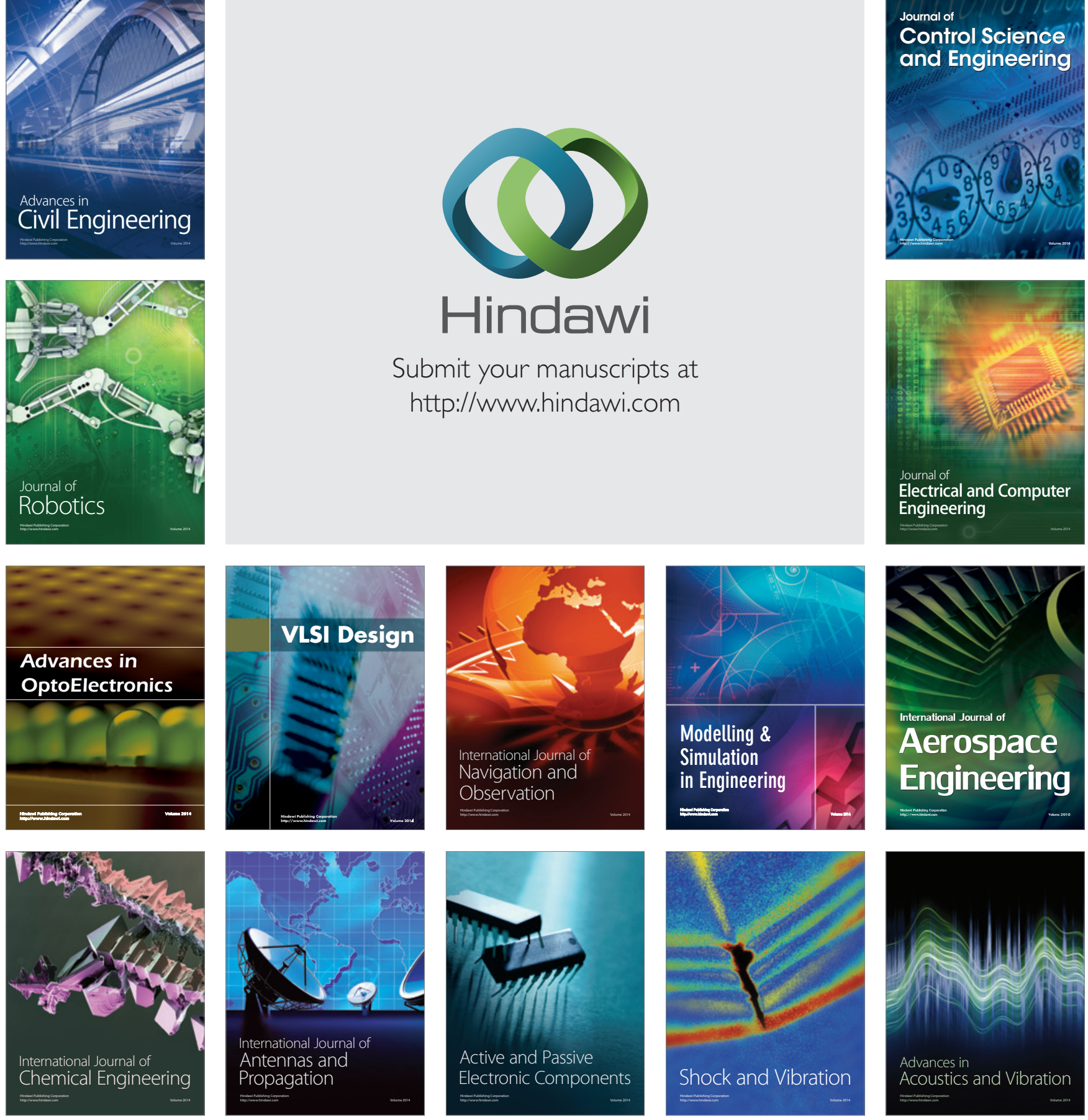